\title{
DIAGNÓSTICO CLINICO DIFERENCIAL ENTRE OCLUSÃO DA ARTÉRIA CARóTIDA INTERNA E DA ARTÉRIA CEREBRAL MÉDIA
}

\author{
ROBERTo MeLaragno * \\ Mario Cinelli JUNIOR ***
}

\author{
Wilson Lufz Sanvito ** \\ ANOI CASTRO CoRDEIRo ****
}

Dois aspectos da patologia da oclusão da artéria carótida interna e da artéria cerebral média já são suficientemente conhecidos para que mereçam maiores considerações.

O primeiro é devido à grande capacidade funcional do círculo de Willis para manter uma circulação colateral suficiente e silêncio sintomatológico durante muito tempo, em grande parte dos casos de obstrução completa da artéria carótida interna. Como corolário dêste fato, compreende-se que a isquemia cerebral, transitória ou não, apenas se processará durante as claudicações dessa circulação colateral e não, necessàriamente, no momento da oclusão da artéria carótida interna ${ }^{12}$. Esta coincidência neurológica apenas se verificará nos casos, raros, em que o círculo de Willis - em virtude de sua constituição anatômica individual, por redução ateroesclerótica de seus ramos comunicantes ou pela inexistência, no momento, de um gradiente mínimo de tensão arterial sistêmica - for insuficiente para garantir uma circulação sangüinea colateral no território do hemisfério abruptamente privado de circulação.

Nos casos de oclusão da artéria carótida interna o maior contingente de sangue proveniente da artéria carótida interna contralateral, através da artéria comunicante anterior, se destinará, em virtude da direção dos vasos, para a artéria cerebral anterior do hemisfério isquemiado. Desta forma, o território de irrigação da artéria cerebral anterior é a área mais protegida pelo círculo de Willis, reconhecidamente o principal sistema de circulação colateral nessas condições. O território de irrigação da artéria cerebral média, quando êsse tronco é obstruído, recebe seu maior contingente de circulação colateral, em nivel pial, por suas anastomoses término-terminais com as demais artérias cerebrais (anterior e posterior). Esse mesmo território, por outro lado, é muito mais favorecido por uma circulação colateral via círculo de Willis nos casos de oclusão da artéria carótida interna. Ainda como corolário dêsses fatos, deriva a grande semelhança dos quadros clínicos de oclusão das artérias carótida interna e cerebral média. Todavia, com o progresso

Trabalho do Serviço de Neurologia (* Chefe; ** Assistente) e do Departamento de Cirurgia Vascular (*** Chefe; **** Médico auxiliar) do Hospital do Servidor Público do Estado de Sāo Paulo, apresentado no IX Congresso Internacional de Neurologia (New York, setembro, 1969). 
da cirurgia cérebro-vascular extracraniana, há freqüentemente razões práticas para se procurar identificar, sobretudo na fase aguda da afecção, a séde da obstrução arterial. Esse reconhecimento, conforme demonstra a experiência de diversos autores e consignada no presente trabalho, é difícil e às vêzes impossivel, exigindo, na maioria dos casos, o emprêgo de angiografia cerebral.

\section{MATERIAL E METODOS}

Este trabalho se baseia no estudo clínico de 61 casos de oclusão da artéria carótida interna e de 23 da artéria cerebral média. Os casos procedem, em sua maioria, do Serviço de Neurologia do Hospital do Servidor Público do Estado de Sāo Paulo e, alguns, da clínica particular de um de nós. Em todos os casos o diagnóstico topográfíco da lesão foi determinado pela angiografia cerebral. Não diferenciamos, nêsse estudo, entre os pacientes com oclusão da carótida interna, aqueles que foram encaminhados à cirurgia vascular daqueles cujo tratamento foi apenas conservador. Separados nossos casos em dois grupos, conforme a sede carotidea ou silviana da obstrução, estudamos, paralelamente e de modo comparativo, o sexo dos pacientes, sua idade, o modo de inicio (apoplectiforme, não apoplético ou progressivo), a incidência ou não de convulsões, cefaléias ou vômitos em fase aguda, o aparecimento de isquemia durante o sono ou vigilia, a existência ou não de ictos anteriores, o estado de consciência após a instalação do processo agudo encefálico. A seguir, comparamos, em ambos os grupos, as caracteriticas topográficas das hemipleigas decorrentes, a tensāo arterial na fase aguda da afecção, a semiologia (palpação e/ou ausculta) das artérias carótidas ao nivel do pescoço, o eletrencefalograma e a oftalmodinamometria. Como o número de nossos casos era ainda relativamente pequeno e, principalmente, porque as diferencas entre os dados encontrados em ambos os grupos se revelaram em geral diminutas, analisamos estatisticamente os resultados, mediante o método do Qui quadrado, ao nível de significância de $5 \% *$.

\section{R E S U L T A D O S}

Sexo - E universalmente reconhecida a predominância da patologia vascular encefálica no sexo masculino, quer se trate de oclusão dos vasos em seu trajeto intracraniano, quer em nível cervical. Em pacientes com oclusão comprovada da artéria carótida interna, registrados na literatura, predomina sempre, no total dos casos, o sexo masculino (87:107 pacientes na casuística de Johnson e Walker ${ }^{8}$; 29:39 na de Paillas e Cristophe ${ }^{13} ; 6: 7$ na de Isch $^{7}$ ). Todos os 7 casos de Feiring ${ }^{4}$ se referiam a homens e, na estatística de Zaclis e col. ${ }^{18}$, a incidência masculina era de $4: 1$. Essa predominância do sexo masculino também parece se verificar nas oclusōes da artéria cerebral média, pràticamente nas mesmas proporções. Em nossa casuística, o sexo masculino foi registrado em $70,5 \%$ dos pacientes com oclusão carotídea e em $69,5 \%$ das obstruções da artéria cerebral média. No estudo de Silverstein e Hollin ${ }^{16}$ essa diferença era menos marcada e, surpreendentemente, eram do sexo masculino apenas 24:50 casos de obstrução carotídea, enquanto que, sôbre 50 casos com oclusão da cerebral média, 37 eram homens.

* Agradecemo ao Dr. Edison Schapochnik a valiosa orientação no tratamento estatístico dos resultados. 
Idade - Como foi mencionado, a idade dos pacientes se refere ao aparecimento da sintomatologia e não, necessàriamení, ao estabelecimento da oclusão; em outros têrmos, refere-se, essencialmente, ao momento da falência da circulação colateral. Por outro lado, a idade dêsses pacientes é paralela à época da formação de placas de ateroma nos vasos que se destinam ao encéfalo. Todavia, como insistem Flora e col. ${ }^{5}$, a idade não é o único fator para a ateroesclerose, pois êsse processo pode ser encontrado em crianças $\mathbf{e}$ até em lactentes. Os grupos etários em que costuma eclodir a sintomatolo-

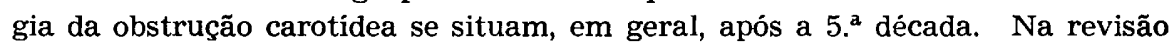
de 107 casos de oclusão carotídea, feita por Johnson e Walker ${ }^{8}$, prepondera o grupo etário entre 30 e 60 anos, variando as idades entre 3 a 71 anos. $\mathrm{Na}$ casuística de Paillas e Cristophe ${ }^{13}$, igualmente, na maioria dos casos, ocorreu entre a $4 .^{\text {e }} 5 .^{\text {a }}$ décadas. Em nosso meio, Zaclis e col. ${ }^{18}$ registraram idades variando entre 10 e 56 anos, predominando a maioria (20:23) entre as idades de 30 a 40 anos. No trabalho de Silverstein e Hollin ${ }^{16}, 40$ dos 50 pacientes com oclusão da artéria cerebral média e 42:50 de obstrução da artéria carótida interna haviam ultrapassado a idade de 50 anos. Em nossa casuística, observamos que, entre as idades de 40 a 70 anos, principalmente entre 50 e 70 anos, situa-se a maior parte dos pacientes. Haveria maior tendência a grupos etários algo mais baixos para a trombose de cerebral média em relação à artéria carótida interna; entretanto, estas cifras carecem de importância estatística.

Modo de inicio - A fenomenologia neurológica inicial poderia, segundo alguns autores, indicar a sede provável do processo oclusivo. Desta forma, as obstruções carotídeas, com maior freqüência do que as da cerebral média, se apresentariam sob forma de pequenos ictos, repetidos e transitórios ("little strokes" ou "transient ischemic accidents"). Em contraposição, os ictos definitivos seriam mais freüentes no grupo das oclusões da artéria cerebral média. Todavia, segundo as estatísticas de Silverstein e Hollin ${ }^{16}$, a incidência de acidentes isquêmicos transitórios foi sensivelmente igual em ambos os grupos (14:50 e 9:50), respectivamente nas oclusões da carótida e da silviana. A existência de ictos prévios em nossa casuística foi também sensivelmente igual em ambos os grupos: $26,2 \%$ e $30,4 \%$. Ainda em nosso material, verificamos o icto apoplético em $16,4 \%$ das tromboses carotídeas e em 30,5\% das obstruções da cerebral média. Entretanto, ictos sem perda imediata da consciência (icto não apoplético) foram registrados em $77,1 \%$ do primeiro grupo e em $56,5 \%$ do segundo. Sintomatologia progressiva foi também registrada em $6,5 \%$ e em $13,0 \%$ de nossos pacientes, respectivamente.

Convulsões, cefaléias e vômitos - A incidência de convulsōes, do tipo generalizado ou localizado, como parte da fenomenologia inaugural, foi significantemente maior, em nossa casuística, no grupo de oclusões da cerebral média em relação ao da carótida interna. Assim, enquanto que nêste último grupo, registramos sòmente em $9,8 \%$ das vêzes, no grupo correspondente à cerebral média, assinalamos $9: 23$, isto é, 39,1\%. Esta cifra é muito maior do que a registrada habitualmente na literatura. Silverstein e Hollin ${ }^{16}$ ape- 
nas assinalaram convulsões em 5 de seus 50 casos de oclusão da artéria cerebral média. Possivelmente, a observação de maior número de casos de oclusões nesta sede poderia diminuir esta porcentagem.

A queixa de cefaléia foi registrada, em nossa casuística, pràticamente com a mesma freqüência nos dois grupos $(31,0 \%$ no da oclusão carotídea e $39,1 \%$ no da artéria cerebral média). Silverstein e Hollin ${ }^{16}$ assinalaram, respectivamente, cefaléias em $20 \%$ e $10 \%$ de seus. casos, em cada grupo. Da mesma forma, em nossa casuística, assinalamos a mesma freqüência de vômitos nos primórdios da afecção $(14,7 \%$ e $13,3 \%$, respectivamente).

Estado de consciência - Observamos, no que se refere ao nosso material, um estado de consciência normal em $77 \%$ das oclusões da carótida contra $61,2 \%$ da cerebral média. Havia torpor em $11,4 \%$ contra $17,3 \%$ e coma em $11,4 \%$ contra $17,3 \%$ respectivamente.

Hemiplegias - Pelos motivos expostos no início dêste trabalho, o território de irrigação da artéria cerebral média é o mais sacrificado pelas oclusões mal compensadas da artéria carótida interna. Desta forma, quer na oclusão dêste vaso, quer na da artéria cerebral média, haverá maior comprometimento da superfície convexa do hemisfério do que de sua parte interhemisférica. Poupado assim, muitas vêzes, o lobo paracentral, a hemiplegia, quando desproporcional, predominará no membro superior em relação ao inferior, quer seja a sede da oclusão na carótida quer na cerebral média. Com efeito, nas 49:50 e 46:50 oclusões da carótida e da cerebral média, na casuística de Silverstein e Hollin ${ }^{16}$, havia hemiplegia e em todos predominava o comprometimento do membro superior. Em nenhum caso, foi observado acometimento isolado do membro inferior. Paralisia supranuclear do facial foi verificada em um mesmo número de casos, em ambos os grupos. Em nossa casuística, observamos hemiplegia com predominância braquial em $55,7 \%$ das oclusões carotideas e em $25,9 \%$ das da artéria cerebral média. Hemiplegias proporcionais foram assinaladas, respectivamente, em $32,6 \%$ e em $\mathbf{7 3 , 8} \%$ dos casos. Possivelmente, essa divergência de nossos dados em comparação com os de Silverstein e Hollin possa depender da diferença de critério de hemiplegia proporcionada. Excepcionalmente, na bibliografia consultada, verificam-se hemiplegias com predominância crural em qualquer dos grupos; em nossa casuística, as registramos em 4,8\% das tromboses da carótida e nenhuma vez nas oclusões da silviana. Em 6\% das obstruções carotídeas, verificadas pela angiografia, não havia qualquer déficit motor.

Tensão arterial - Independentemente da sede da oclusão, mas pràticamente na mesma proporção, a hipertensão arterial foi registrada na maior parte das vêzes $(65,7 \%$ e $73,9 \%$ respectivamente, nas oclusões da carótida e da cerebral média).

Palpação e ausculta das carótidas - A palpação das artérias carótidas no pescoço fornece resultados sugestivos para o diagnóstico da sede cervical da oclusão dêsse vaso apenas quando se verifica ausência de pulsação do 
mesmo. A reciproca, entretanto, não é necessàriamente verdadeira, pois em casos comprovados de oclusão carotídea, pode existir uma percepção táctil do pulso carotídeo, quer pela sede mais craniana da obstrução, ao nível do sifão, quer pela sensação digital da pulsação da artéria carótida externa (às vêzes hiperpulsátil), sendo pràticamente indiferençáveis, entre êsses vasos, os batimentos de ambos os lados ${ }^{14}$. Por outro lado, a ausculta de sôpros, principalmente ao nível da bifurcação da artéria carótida primitiva deve ser valorizada como sinal sugestivo de ateroma ou obstrução da artéria carótida interna. Todavia, cuidados especiais devem ser tomados a fim de não se supervalorizar a existência dêsses sôpros que podem ser notados em indivíduos do mesmo grupo etário sem manifestações cérebro-vasculares ou, mesmo, em individuos jovens, sem qualquer patologia ${ }^{11}$. McDowell, em um grupo de 37 pacientes com sôpros, realizou angiogramas em 33 e verificou que em 25 , isto é, em $75 \%$, havia uma estenose arterial significante onde o sôpro era auscultado; por isso, considerou o sôpro como sinal válido, indicativo de estenoses arteriais extracranianas. O valor relativo, apenas sugestivo dos sôpros nas estenoses carotídeas é também assinalado por Burton ${ }^{2}$ e Crevasse $^{3}$. Por outro lado, uma estenose carotídea pode, evidentemente, coexistir com oclusão completa da artéria cerebral média, podendo sugerir uma patologia exclusiva no primeiro dêsses vasos. Em nosso material registramos que, em $44,2 \%$ dos pacientes com oclusões comprovadas da carótida, não foram encontradas alteraçб́es da pulsação ou à ausculta da carótida. Em porcentagem muito maior ( $91,2 \%$ dos casos) de oclusão da artéria cerebral média, também não se registraram alterações da pulsação ou da ausculta carotidea. Pelo contrário, em mais de metade dos casos de patologia carotídea $(52,4 \%)$ observaram-se alterações à pulsação ou à ausculta dêsse vaso em nível cervical. Essas anormalidades foram observadas ùnicamente em 8,6\% dos pacientes com oclusão da artéria cerebral média. Estes dados comparativos assumem significância estatística em nossa casuística.

Eletrencefalograma - Quando êste exame foi realizado, verificamos sua anormalidade em $76 \%$ dos pacientes com oclusões carotídeas contra $88,9 \%$ dos casos de obstrução silviana. Os traçados foram realizados em condições basais de repouso, sensibilizando-se a prova pela hiperpnéia e pela foto-estimulação. Provàvelmente, a porcentagem de anormalidades seria maior se sistemàticamente fosse acrescentada a essas provas de sensibilização a compressão alternada das carótidas, conforme recomendam Taillandier e col. ${ }^{17}$ e como, atualmente, estamos realizando como rotina.

Oftalmodinamometria - Após emergir do seio cavernoso, o primeiro ramo emitido pela artéria carótida interna, em seu trajeto supraclinóide, é a artéria oftálmica de onde se origina a artéria central da retina. Como, desde a bifurcação em nivel cervical até a origem da artéria oftálmica, a artéria carótida interna não emite nenhum outro ramo, podemos admitir que a cabeça de pressão da artéria carótida interna é fielmente refletida na pressão da artéria oftálmica. Assim, a determinação da pressão da artéria central da retina, ramo da oftálmica, pode ser um indice valioso para o conhecimento 
da pressão da carótida. Desta forma, uma estenose ou trombose dêste último vaso costuma se refletir na pressão da artéria central da retina; a oftalmodinamometria revelará, nessa eventualidade, valores diastólicos e sistólicos menores do que no lado oposto, constituindo essa assimetria um sinal sugestivo de processo obstrutivo cervical da carótida interna. Se a sede da obstrução residir na cerebral média - ramo emitido distalmente à artéria of tálmica -, não há motivos para se registrarem assimetrias oftalmodinamométricas. Hollenhorst ${ }^{6}$, em 228 casos de doença oclusiva da artéria carótida, observou 162 vêzes (61\% dos casos) a pressão retiniana diastólica no lado da artéria carótida acometida sensivelmente reduzida. Entretanto, pressōes mais altas foram medidas no lado hemiplégico, isto é, na carótida normal, em 9 casos (4\%); os niveis eram iguais em ambos os lados em 57 casos (25\%). Como regra a posição deitada acentua as diferenças entre ambos os lados. Bettelheim ${ }^{1}$ também registrou um caso de trombose da artéria carótida interna em nivel cervical com predomínio paradoxal da pressão da artéria oftálmica homolateral; como explicação para êste caso, admite a existência de uma circulação colateral aumentada entre ramos da artéria carótida externa e a artéria oftálmica e, concomitantemente, propagação do trombo da artéria carótida interna, distalmente à saída da artéria oftálmica. Em nosso material, nos casos em que o exame foi realizado, observamos nas oclusões da carótida a anormalidade oftalmodinamométrica em 70\% dos casos, enquanto que, nos poucos casos de oclusão da cerebral média em que o exame foi realizado, nenhuma vez observamos assimetrias entre os níveis pressóricos das artérias centrais da retina.

Embora alguns autores façam menção à maior incidência de distúrbios sensitivos e de alterações nos campos visuais em determinadas sedes da oclusão, não computamos êsses dados, em virtude da falta de cooperação de muitos pacientes, quer pelos distúrbios da consciência, quer pela afasia coexistente. Convém assinalar, entretanto, que Silverstein e Hollin ${ }^{16}$ registraram, em sua casuística, incidências sensivelmente iguais de hemianestesia e hemianopsia nos grupos com oclusão da artéria carótida interna e da artéria cerebral média. Resultados discordantes, todavia, são referidos por Louis e Lewis ${ }^{10}$ que analisaram 16 pacientes com oclusão da carótida e 9 com oclusão da artéria cerebral média em que foi possivel um estudo da sensibilidade. Por outro lado, reuniram na literatura a observação de 196 pacientes com oclusão da carótida e 19 da artéria cerebral média, registrando, então, muito maior freqüência de alteraçōes da sensibilidade na obstrução da artéria cerebral média e do sifão carotídeo do que na trombose cervical da carótida interna. Assim, notaram em 5 de seus próprios casos de oclusão da carótida interna, assim como em $25 \%$ daqueles cujas observações recolheram, uma hemianestesia contralateral. Em contraposição, alterações da sensibilidade se evidenciaram em 8 de seus 9 pacientes com oclusão da cerebral média e em $90 \%$ dos casos da literatura. Obviamente, a existência de distúrbios visuais unilaterais, prévios ou atuais, sobretudo acompanhados de atrofia óptica e sediados no lado oposto à hemiplegia, depõem decisivamente para o diagnóstico de trombose carotídea; todavia, estas hemiplegias alternas optopira- 
midais são excepcionais no conjunto dos casos de oclusões carotídeas. Também pode sugerir esta sede de obstrução a verificação de uma sindrome de Claude Bernard-Horner ou, mesmo, de simples miose.

\section{R E S U M O}

Foi feito um estudo comparativo entre o quadro clínico inicial de 61 casos de oclusão da artéria carótida interna e o de 23 casos de oclusão da artéria cerebral média, diagnosticados pela angiografia cerebral e/ou pela necrópsia em pacientes submetidos ou não à cirurgia vascular. Os autores comparam a idade dos pacientes, o sexo, o modo de início da afecção, a existência ou não de convulsões e/ou cefaléias, a ocorrência do acidente cerebral durante o sono ou em vigília, a existência de ictos prévios, os níveis de pressão arterial, o grau de consciência, a fôrça muscular, os achados eletrencefalográficos, a palpação e ausculta das carótidas em nivel cervical. Os resultados são demonstrados em indices percentuais, pelos quais os autores inferem que não há significância estatística nesses elementos com três exceções : a maior ocorrência de convulsões nas oclusões da artéria cerebral média em relação às da carótida, a oftalmodinamometria e a sintomatologia carotídea cervical. A oftalmodinamometria revela valores significantemente menores nas pressões da artéria central da retina, no mesmo lado da trombose da carótida em $70,0 \%$ dos casos, enquanto que medidas normais e simétricas verificaram-se em todos os casos de oclusão da artéria cerebral média em que o exame foi realizado. No que concerne a sinais arteriais no pescoço, havia anormalidades palpatórias e auscultatórias em 52,4\% dos pacientes com trombose da carótida e em 8,6\% dos casos com oclusão da cerebral média. Concluem os autores, portanto, que apenas a angiografia cerebral permite um diagnóstico seguro entre ambas as sedes da oclusão.

\section{S U M M A R Y}

A comparative symptomatological study of internal carotid artery occlusion and middle cerebral artery occlusion

A comparative study of the early clinical picture in 61 cases of internal carotid artery occlusion and 23 cases of middle cerebral artery occlusion, diagnosed by cerebral angiography and/or necropsy in patients who were either submitted to surgery or not operated upon is reported. The authors had compared the patients' age and sex, onset of the disease, ocurrence of convulsions and/or headache, onset during sleep or waking, the pre-existence of strokes, blood pressure levels, degree of consciousness, muscular strength, electroencephalography tracings, palpation and auscultation of the cervical carotid arteries. The results of the study did show that there is no statistical significative difference in these data except for 3 of them: greatest incidence of convulsions in middle cerebral artery occlusion, ophtalmodynamometry and cervical carotid symptomatology. Ophtalmodynamometry reveals significantly lower values for retinal central artery pressures on the same 
side as the carotid thrombosis in $70.0 \%$ of the cases, while normal and symmetrical measurements appear in all cases of middle cerebral artery occlusion. With reference to arterial signs in the neck, there were palpatory and auscultatory abnormalities in $52.4 \%$ of the patients with carotid thrombosis and in $8.6 \%$ of the cases of middle cerebral artery occlusion.

\section{$R$ E F E R N C I A S}

1. BETTELheim, H. - Ungewöhnliches Verhalten des Blutdruckes der Arteria ophtalmica beim Verschluss des Halsteils der Arteria carotis interna. Albrecht v. Graefes Arch. Ophtalm. 168:175, 1965.

2. BURTON, R. C. - Discussão do relatório de McDowell e col. ${ }^{11}$.

3. CREVASSE, L. - Carotid artery murmurs. Clinical and pathophysiologic correlation. Neurology (Minneapolis) 11 (parte II):100, 1961.

4. FEIRING, E. H. - Spontaneous occlusion of the internal carotid artery. Neurology (Minneapolis) 4:405, 1954.

5. FLORA, G. C.; BAKER, A. B. \& KLASSERN, A. - Age and cerebral atherosclerosis. J. Neurol. Sc. 6:357, 1968.

6. HOLLENHORST, R. H. - Cerebrovascular diseases: neuroophtalmologic aspects. Transaction of the Fifth Conference under the auspices of The American Neurological Ass. and The American Heart Ass., New Jersey, 1966.

7. ISCH, F. - Essal clinique sur l'hémiplégie par thrombose de la carotide interne ou de ses branches. Thèse, Strasbourg, 1950.

8. JOHNSON, H. C. \& WALKER, A. E. - The anglographic diagnosis of spontaneous thrombosis of the internal and common carotid arteries. J. Neurosurg. 8:631, 1951.

9. LHERMitTe, F.; GAUTIER, J. C.; DEROUESME, L. \& GUIRAUD, B. - Ischemic accidents in the middle cerebral artery territory. Arch. Neurol. (Chicago) 19:248, 1968.

10. LOUIS, S. \& LEWIS, B. - Sensory disorders in occlusion of internal carotid and middle cerebral arteries. Neurology (Minneapolis) 13:693, 1963.

11. MeDOWELL, F.; RENNIE, L. \& EJRUP, B. - Arterlal bruit in cerebrovascular disease. Transactions of the Fifth Conference under the auspices of The American Neurological Ass. and The American Heart Ass., New Jersey, 1966.

12. MELARAgNO FILHO, R. - Afeccões Vasculares Cerebrais. Diagnóstico e Tratamento. Livraria Luso-Espanhola e Brasileira Editora, São Paulo, 1959.

13. PAIllas, J. \& CHRISTOPHE, L. - Les Thromboses de la Carotide Interne et ae ses Branches. Masson et Cie., Paris, 1955.

14. ROSEGAY, H. - Limited value of carotid pulse in diagnosis of internal carotid thrombosis. Neurology (Minneapolis) 6:143, 1956.

15. SIEKERT, R. - Incipient or impeding strokes: focal intermittent insuficiency of ischemic attacks. Transactions of The Fifth Conference under the auspices of The American Neurological Ass. and The American Heart Ass., New Jersey, 1966.

16. SILVERSTEIN, A. S. \& HOLLIN, S. - Internal carotid vs. middle cerebral artery occlusions. Clinical differences. Arch. Neurol. (Chicago) 12:468, 1965.

17. TAILLANDIER, P.; REVOL, M.; QUOEX, F.; GERIN, P. \& COURJON, J. Etude polygraphique des obstructions carotidiènnes et sylviènnes: interét des compressions carotidiènnes controlées. Rev. Neurol. (Paris) 119:308, 1968.

18. ZACLIS, J.; CRUZ, O. R. \& ALMEIDA, G. G. M. - Obstrução das artérias carótidas e das principais artérias cerebrais. Arq. Neuro-Psiquiat. (São Paulo) $14: 158,1956$. 